\title{
VIBRAÇÃO E RUÍDO EM UM TRATOR AGRÍCOLA SUBMETIDO A DIFERENTES SUPERFÍCIES DE ROLAMENTO
}

\author{
Patrícia Adriana Marques de Andrade (1); \\ Vinícius Paludo (2) \\ Jefferson Sandi (3) \\ Murilo Basttistuzzi Martins (4) \\ João Eduardo Guarnetti dos Santos (5)
}

(1) Universidade Estadual Paulista "Julio de Mesquita Filho" - Faculdade de Ciências Agronômicas FCA, Especialista em Engenharia de Segurança do Trabalho.

patrícia_pama@hotmail.com

(2) Universidade Estadual Paulista "Julio de Mesquita Filho" - Faculdade de Ciências Agronômicas FCA, Mestre em Energia na Agricultura.

vipaludo@gmail.com

(3) Universidade Estadual Paulista "Julio de Mesquita Filho" - Faculdade de Ciências Agronômicas FCA, Mestre em Energia na Agricultura.

$$
\text { jffsandi@gmail.com }
$$

(4) Universidade Estadual Paulista "Julio de Mesquita Filho" - Faculdade de Ciências Agronômicas FCA, Mestre em Energia na Agricultura.

mbm_martins@hotmail.com

(5) Universidade Estadual Paulista "Julio de Mesquita Filho" - Faculdade de Engenharia FEB Bauru, Livre Docente.

$$
\text { guarneti@feb.unesp.br }
$$

\footnotetext{
RESUMO

O trabalho teve por objetivo medir e avaliar vibração e ruído em um trator com e sem implemento deslocando-se em três diferentes superfícies de rolamento. Utilizou-se um trator agrícola 4X2 com tração dianteira auxiliar (TDA) de $197 \mathrm{cv}(144,8 \mathrm{~kW})$, os dados de vibração foram obtidos por um registrador e medidor de vibração HVM 100, conectado a um sensor de aceleração piezelétrico de três eixos $(X, Y$ e $Z$ ) e o ruído na cabine do trator foi obtido utilizando um decibelímetro. $O$ ruído máximo interno da cabine permite que o operador desempenhe uma jornada de trabalho de 8 horas. Somente foram geradas condições insalubres na pista padronizada de ensaio de vibração independentemente da presença de implemento acoplado ao sistema de três pontos do trator.
} 


\begin{abstract}
The study aimed to measure and evaluate vibration and noise on a tractor with and without attachment moving on three different surfaces bearing. Used a $4 \times 2$ tractor with auxiliary front wheel drive (TDA) to $197 \mathrm{hp}(144.8 \mathrm{~kW})$, the vibration data were obtained by a recorder and vibration meter HVM 100, connected to a piezoelectric acceleration sensor of three axes ( $X, Y$ and $Z)$ and the noise in the tractor cab was obtained using a decibel meter. The maximum internal cabin noise allows the operator to perform an 8 hour working day. Only it was generated unsanitary conditions in the standardized test track vibration regardless of the presence of the implement attached to the tractor three-point system
\end{abstract}

\title{
1. INTRODUÇÃO
}

Trabalhadores rurais são expostos a vários riscos ocupacionais durante sua jornada de trabalho, como os mecânicos (decorrentes do manuseio de máquinas), físicos (radiação solar, frio, calor e o ruído), químicos (poeira, fuligem, gases e agroquímicos), biológicos (animais peçonhentos e microrganismos), além dos relacionados a organização do trabalho (ROSEIRO, TAKAVANAGUI, 2004; BRAUNBECK et al., 2012).De acordo com Tosin (2009), com o aumento da utilização de máquinas agrícolas as condições de segurança do operador não tiveram devida correspondência, de forma que o condutor do trator se torna um dos profissionais mais expostos a fatores insalubres.

Os parâmetros ergonômicos em máquinas agrícolas não se restringem somente a disposição dos comandos, o posicionamento e características do assento, espaço interna da cabine, presença de sistema de ventilação, etc; quando se refere à avaliação de parâmetros ergonômicos incluem-se na avaliação também os agentes insalubres como ruído e vibração que são produzidos pela máquina (DOIMO 2015).

Mesmo com a grande importância do setor agrícola no Brasil, parte dos tratores produzidos no país não são projetados para fornecer uma adequada relação homem-máquina (conforto, segurança, facilidade de acessos, disposição de comandos, etc), sendo a concepção do projeto voltada para a otimização dos parâmetros operacionais e desempenho, resultando em máquinas que expõem o operador às condições climáticas, ao ruído e a vibração (SANTOS et al. 2014). Dessa forma, embora no Brasil exista a obrigatoriedade quanto ao seguimento das NRs e NBRs na inspeção de máquinas, nem sempre as mesmas são seguidas da forma exigida, resultando na reduçãoda qualidade ocupacional dos operadores das máquinas (Baesso et al. 2015).

As máquinas agrícolas, durante seu funcionamento, geram vibrações mecânicas que são transmitidas por toda a sua estrutura e para o ambiente que a rodeia. Quando estas ocorrem em níveis indesejados e por períodos de exposição mais longos que o suportável, podem causar problemas de saúde aos indivíduos expostos às mesmas (SANDI 2015).

Para Lanças et al. (2009), as vibrações estão diretamente ligadas ao afastamento por doença do trabalho no país. Estas vibrações ocasionam grande desconforto e aumentam a fadiga do trabalhador, podendo-se classificar as mesmas de acordo com a região do corpo atingida em: vibrações de corpo inteiro, comuns em atividades de transporte, tais como caminhão, trator, empilhadeira, ônibus, etc., e vibrações de extremidades que afetam principalmente mãos e braços.

As vibrações mecânicas originadas tanto no funcionamento da máquina quanto pela rugosidade da superfície de deslocamento se tornam problemáticas quando a frequência de partes do corpo humano (por exemplo, o tronco vibra a uma frequência de 4 a $8 \mathrm{~Hz}$ ), acaba se aproximando a do trator $(1-7 \mathrm{~Hz})$, o que pode elevar as chances de problemas de saúde no operador (ZEHSAZ et al., 2011). 
Dessa forma, são necessários que estudos sejam realizados constantemente no intuito de quantificá-las, para que, se necessário, possam ocorrer adaptações dos maquinários ou legislações. (SILVA, MIRANDA e VIANA 2015).

Por sua vez, o som é definido como variação da pressão atmosférica dentro dos limites de amplitude e banda de frequências. No entanto, não são todas as flutuações de pressão que produzem a sensação de audição quando atingem o ouvido humano (GERGES, 2000).

O ouvido humano suporta até $85 \mathrm{~dB}$ por volta de 8 horas exposto ao ruído sem qualquer desconforto, mas ao colocarmos o ouvido a ruídos que ultrapassam a máxima exposição diária permissível, a faixa audível de um ser humano pode sofrer perda em um determinado prazo e muitas vezes essas perdas acabam sendo irreparáveis. Porém, o ruído é o agente físico insalubre presente em mais de $90 \%$ das atividades laborativas existentes. $\mathrm{Na}$ operação de um trator é o principal risco ao qual o operador está exposto (TOSIN, 2009).

Para ABRAHÃO et al. (2009) algumas situações produzem níveis elevados de ruídos provocando vários efeitos negativos não somente para a saúde das pessoas, mas também para o desempenho do trabalhador.

Dessa forma, sendo o trator uma máquina que durante seu funcionamento apresenta altos valores de ruído e vibrações mecânicas devido as suas características construtivas e condições encontradas no local de construção, este trabalho teve como objetivo medir e avaliar estes dois fatores em um trator com e sem implemento deslocando-se em três diferentes superfícies de rolamento.

\section{MATERIAL E MÉTODOS}

O ensaio foi realizado na Faculdade de Ciências Agronômicas da Universidade Estadual Paulista "Júlio de Mesquita Filho", Campus de Botucatu - SP (22 $51^{\prime}$ 'S e $\left.48^{\circ} 25^{\prime} \mathrm{W}\right)$, a uma altitude de 760 metros.

O experimento foi realizado por meio de operações executadas em três diferentes superfícies, sendo uma pista de vibração normatizada, construída em madeira em acordo com a norma ISO 5008 (2002). Os degraus foram dispostos ao longo dos dois trechos de deslocamento obedecendo aos critérios estabelecidos pela norma, uma pista de concreto com 200 metros de comprimento e 4 metros de largura construída segundo a Norma OECDCode 2 (2008), totalizando $800 \mathrm{~m}^{2}$ de área útil e declividade de $1 \%$ no sentido do comprimento, e uma pista de solo mobilizado com 400 metros de comprimento e 20 metros de largura totalizando $8.000 \mathrm{~m}^{2}$ de área e declividade de $1 \%$ no sentido do comprimento. $\mathrm{O}$ solo da pista foi classificado segundo a Embrapa (1999), como Nitossolo Vermelho Distroférrico com relevo plano e textura argilosa.

Foi utilizado um trator agrícola 4X2 com tração dianteira auxiliar (TDA) de 197 cv (144,8 $\mathrm{kW}$ ), equipado com cabine modelo padrão dotada de sistema de amortecimento oferecida pelo fabricante. Foram utilizados pneus traseiros 30.5L/32 LS-2 com pressão de inflação de 41,37 kPa e pneus dianteiros 420/85-82 com pressão de inflação de 55, 16 kPa.

Também foi empregado um distribuidor de fertilizantes a lanço com acoplagem pelo sistema de três pontos do trator da marca SEMBRA modelo $1300 \mathrm{PAH}$ com capacidade de carga para 1800 quilos, o qual era acionado pela tomada de potência (TPD) do trator agrícola.

Para obtenção dos dados de vibração utilizou-se um registrador e medidor de vibração HVM 100 da marca Larson Davis, conectado a um sensor de aceleração piezelétrico de três eixos (X, Y e Z) modelo SEN027-CBL devidamente calibrado. A medição da vibração foi realizada utilizando-se o registrador e medidor de vibração modelo HVM-100 para levantamento dos valores de aceleração de corpo inteiro. O acelerômetro foi fixado sobre o assento do operador de acordo com a NHO 09 (eixo x - frente/trás; eixo y - direita/esquerda; e eixo z para cima/para baixo). Para evitar a movimentação do disco, este foi fixado ao assento por meio de fita adesiva. O aparelho coletor dos dados de vibração foi ajustado de modo a 
coletar os parâmetros referentes à vibração no corpo todo, presentes nos três eixos e o intervalo de aquisição dos dados. Foram obtidos os valores de valor da dose de vibração (VDV) e projeção de dose para 8 horas (A8).

O valor da dose de vibração é obtido a partir do método de dose de vibração à quarta potência determinada na direção "i", onde "i" corresponde aos eixos ortogonais " $x$ ", " $y$ " ou " $z$ ", expresso em $\mathrm{m} \mathrm{s}^{-1,75}$ (Equação 1). Nas normas internacionais adota-se o valor dose de vibração como sendo o maior valor de dose de vibração obtido entre os três eixos ortogonais, enquanto a Norma NHO 09 (2013) obtém este valor através do conceito de valor dose resultante (VDVR).

$\mathrm{VDV}: \sqrt[4]{\int_{0}^{t}[a i(t)]^{4} d t}=\left[\mathrm{m} \mathrm{s}^{-1,75}\right]$

[Eq. 01]

Onde:

aj(t) = aceleração instantânea ponderada em frequência;

$\mathrm{t}=$ tempo de duração da medição.

A projeção $A(8)$, é um método de avaliação que gera um valor médio de vibração ajustado para representar a dose de uma jornada de trabalho de oito horas, pois no banco do operador os valores se mantem de forma relativamente estável durante este período (SCARLETT et al., 2007). A projeção A(8) é dada pela Equação 2.

$\mathrm{A}(8): a p \sqrt{\frac{t}{t_{0}}}$

Onde:

$a p=$ é a aceleração ponderada, combinada nos três eixos, equivalente em $\mathrm{m} \mathrm{s}^{-2}$;

t0= é uma duração de referência de 8 horas.

Conjuntamente com a aquisição de dados de vibração em corpo inteiro, foi avaliado o nível de ruído na cabine do trator, sendo utilizado um decibelímetro de medição de nível sonoro, digital e portátil da marca INSTRUTHERM - modelo DEC $300 \mathrm{com}$ escala de 40 a $130 \mathrm{~dB}$, o qual foi configurado para coletar os dados na faixa $A$ no modo rápido, posicionado ao lado do operador, onde foi registrado o valor de máximo e mínimo do ruído do trator agrícola dentro da cabine.

Para todos os tratamentos foi utilizada a mesma velocidade de deslocamento através da fixação de uma rotação do motor e marcha de trabalho. A velocidade média de deslocamento foi obtida através da Equação 1.

$v=d \cdot t^{-1}$

[Eq. 03]

Onde:

$\mathrm{v}=$ velocidade de deslocamento $\left(\mathrm{m} \mathrm{s}^{-1}\right)$;

$\mathrm{d}=$ distância percorrida pelo conjunto $(\mathrm{m})$;

$\mathrm{t}=$ tempo de deslocamento (s). 
Para avaliação do nível de insalubridade vibracional utilizou-se os parâmetros estabelecidos na Norma de Higiene Ocupacional NHO09 (Tabela 1), e para a insalubridade causada pelo ruído foi empregada à tabela disponibilizada pela Norma Regulamentadora NR15 (Tabela 2).

Tabela 1. Critério de julgamento e tomada de decisão para exposição vibracional de corpo inteiro.

\begin{tabular}{cccc}
\hline $\begin{array}{c}\text { aren } \\
\left(\mathbf{m ~ s}^{-2}\right)\end{array}$ & $\begin{array}{c}\text { VDVR } \\
\left(\mathbf{m ~ s}^{-1,75}\right)\end{array}$ & $\begin{array}{c}\text { Consideração } \\
\text { Técnica }\end{array}$ & Atuação Recomendada \\
\hline 0 a 0,5 & 0 a 9,1 & Aceitável. & No mínimo manutenção da condição existente. \\
\hline$>0,5 a<$ & $>9,1$ a $<$ & Acima do nível de \\
0,9 & 16,4 & ação. & No mínimo adoção de medidas preventivas. \\
\hline 0,9 a 1,1 & $\begin{array}{c}16,4 \text { a } 21 \\
\text { Região de incerteza. }\end{array}$ & $\begin{array}{c}\text { Adoção de medidas preventivas e corretivas } \\
\text { visando à redução da exposição diária. }\end{array}$ \\
\hline $\begin{array}{c}\text { Acima de } \\
1,1\end{array}$ & $\begin{array}{c}\text { Acima de } \\
21\end{array}$ & $\begin{array}{c}\text { Acima do limite de } \\
\text { exposição. }\end{array}$ & Adoção imediata de medidas corretivas. \\
\hline
\end{tabular}

Fonte: Norma de Higiene Ocupacional 09 - NHO 09 (2013).

Tabela 2. Máxima exposição diária em relação ao nível de ruídos sem proteção auricular.

\begin{tabular}{cccc}
\hline $\begin{array}{c}\text { Nível de ruídos } \\
\text { dB (A) }\end{array}$ & $\begin{array}{c}\text { Máxima exposição diária } \\
\text { permissível }\end{array}$ & $\begin{array}{c}\text { Nível de ruídos } \\
\mathbf{d B}(\mathbf{A})\end{array}$ & $\begin{array}{c}\text { Máxima exposição diária } \\
\text { permissível }\end{array}$ \\
\hline 85 & 8 horas & 98 & 1 hora e 15 minutos \\
86 & 7 horas & 100 & 1 hora \\
87 & 6 horas & 102 & 45 minutos \\
88 & 5 horas & 104 & 35 minutos \\
89 & 4 horas e 30 minutos & 105 & 30 minutos \\
90 & 4 horas & 106 & 25 minutos \\
91 & 3 horas e 30 minutos & 108 & 20 minutos \\
92 & 3 horas & 110 & 15 minutos \\
93 & 2 horas e 40 minutos & 112 & 10 minutos \\
94 & 2 horas e 15 minutos & 114 & 8 minutos \\
95 & 2 horas & 115 & 7 minutos \\
96 & 1 hora e 45 minutos & & \\
\hline Fonte: Norma Regulamentadora (NR 15) - Atividades e operações Insalubres, 1978.
\end{tabular}

Os tratamentos consistiram no emprego de 3 pistas de ensaios pela presença/ ausência de implemento acoplado ao trator, totalizando 6 tratamentos. Foram coletadas 3 repetições por tratamento, gerando um total de 18 unidades amostrais. Os dados coletados foram analisados através do teste de separação de médias de teste de Tukey $(p>0,05)$ com auxílio do software Sisvar 5.3 (Build 77).

\section{RESULTADOS E DISCUSSÃO}

O ruído interno máximo medido com a cabine do trator fechada durante os ensaios sofreu influência tanto do tipo de superfície de deslocamento quanto da presença/ausência do implemento (Tabela 3). Os maiores valores foram encontrados no tratamento sem implemento em deslocamento sobre pista de concreto (sendo este o tratamento com maior ruído entre todos), e no tratamento com implemento em deslocamento sobre a pista padronizada para ensaio de vibração. 
Tabela 3. Ruídos máximos internos (dB) obtidos com o trator operando com e sem implemento em três condições superficiais distintas.

\begin{tabular}{cccc}
\hline & \multicolumn{3}{c}{ Superfície } \\
\hline Implemento & Concreto & Solo Mobilizado & Pista de Vibração \\
\hline Sem & $83,93 \mathrm{Aa}$ & $76,93 \mathrm{Ab}$ & $77,86 \mathrm{Ab}$ \\
Com & $76,63 \mathrm{Bb}$ & $76,43 \mathrm{Ab}$ & $79,43 \mathrm{Aa}$ \\
\hline F de Implemento & $12,04^{* *}$ & \\
F de Pista & $12,09^{* *}$ & \\
F de Implemento x Pista & $20,27^{* *}$ & \\
CV (\%) & 1,61 & \\
DMS Linha & 2,75 & \\
DMS Coluna & 2,25 & \\
\hline
\end{tabular}

Médias seguidas de mesma letra maiúscula não diferem entre si na coluna e mesma letra minúscula não diferem entre si na linha pelo teste Tukey $(p \leq 0,05)$. ns: não significativo pelo teste $\mathrm{F}$ ao nível de $5 \%$ de probabilidade. * Significativo pelo teste $\mathrm{F}$ ao nível $5 \%$ de probabilidade. ${ }^{*}$ Significativo pelo teste $\mathrm{F}$ ao nível de $1 \%$ de probabilidade.

Quando os valores de ruídos ultrapassam a máxima exposição diária permissível, muitas vezes é necessário verificar as medidas de controle de ruído, podendo ser consideradas basicamente três: na fonte, na trajetória e no trabalhador. Se as medidas de controle na fonte e na trajetória não foram possíveis ou quando elas não foram suficientes, é indicada a utilização de EPI (Equipamento de Proteção Individual), ou controlar o limite de tempo de exposição do trabalhador, através de rodízio de funcionários nas atividades.

Em todas as situações avaliadas os valores de ruído máximo não ultrapassaram o limite de $85 \mathrm{~dB}$ estabelecido pela NR15, permitindo que o operador possa trabalhar em jornada de 8 horas diárias nas condições ensaiadas com a cabine fechada. Baesso et al. (2015), também obteve valores de ruído interno abaixo de $85 \mathrm{~dB}$ para tratores com cabine original de fábrica, atribuindo estes valores ao bom projeto e construção deste componente.

Apesar da eficiência da cabine em isolar o operador dos ruídos externos, é importante que o mesmo tenha seus EPIs disponíveis, pois segundo Pimenta Junior et al. (2012), independente do ambiente onde será realizada a operação com trator agrícola, é imprescindível que qualquer trabalhador, seja ele o operador ou funcionário auxiliar, para desempenhar atividades fora da cabine a menos de cinco metros em relação à posição central do trator deve fazer uso de EPI.

Os resultados de valor de dose de vibração encontrados para a pista padronizada de ensaio de vibração indicam que para operar neste cenário seriam necessários cuidados e medidas para redução da vibração incidente sobre o operador (Tabela 4). Nas demais pistas, os valores encontrados se encontram abaixo do nível de ação, indicando que o operador pode realizar jornadas diárias de 8 horas de trabalho nas quatro condições de operação (nas duas pistas com ou sem implemento). A cooperação benéfica do implemento na atenuação dos valores de vibração é observada quando o trator se desloca sobre as pistas com superfícies irregulares. 
Tabela 4. Valores de dose de vibração $\left(\mathrm{m} \mathrm{s}^{-1,75}\right)$ obtidos com o trator operando com e sem implemento em três condições superficiais distintas.

\begin{tabular}{cccc}
\hline & \multicolumn{3}{c}{ Superfície } \\
\hline Implemento & Concreto & Solo Mobilizado & Pista de Vibração \\
\hline Sem & $1,27 \mathrm{Ac}$ & $2,93 \mathrm{Ab}$ & $10,63 \mathrm{Aa}$ \\
Com & $1,20 \mathrm{Ac}$ & $2,20 \mathrm{Bb}$ & $9,67 \mathrm{Ba}$ \\
\hline F de Implemento & & $112,36^{* *}$ & \\
F de Pista & $9993,00^{* *}$ & \\
F de Implemento x Pista & $23,56^{* *}$ & \\
CV (\%) & 2,53 & \\
DMS Linha & 0,257 & \\
DMS Coluna & 0,209 &
\end{tabular}

Médias seguidas de mesma letra maiúscula não diferem entre si na coluna e mesma letra minúscula não diferem entre si na linha pelo teste Tukey $(p \leq 0,05)$. ns: não significativo pelo teste $F$ ao nível de $5 \%$ de probabilidade. ${ }^{*}$ Significativo pelo teste $\mathrm{F}$ ao nível $5 \%$ de probabilidade. ${ }^{*}$ Significativo pelo teste $\mathrm{F}$ ao nível de $1 \%$ de probabilidade.

O VDV representa a exposição cumulativa da exposição a vibração em um dia de trabalho, sendo mais indicado para avaliação do efeito da vibração sobre o corp humano em jornadas maiores que 8 horas por representar de forma mais adequada os solavancos e choques que ocorrem durante o trabalho (SCARLETT et al., 2007).

Apenas a pista de vibração gerou uma condição de insalubridade, pois gerou valores que ficaram acima do nível de ação $\left(9,1 \mathrm{~m} \mathrm{~s}^{-1,75}\right)$ e abaixo da região de incerteza $\left(16,4 \mathrm{~m} \mathrm{~s}^{-1,75}\right)$, devendo-se assim adotar um conjunto de medidas preventivas para amenizar os efeitos da vibração sobre o operador como redução da velocidade de trabalho, verificação da correta regulagem e posição do assento para operador, evitar sempre que possível superfícies muito irregulares e adoção da correta condição operacional da máquina para cada situação de trabalho.

Segundo Balbinot (2001), quando o valor de $8,5 \mathrm{~m} \mathrm{~s}^{-1,75}$ é excedido existe a possibilidade de desconforto médio, pois segundo o mesmo, este parâmetro de avaliação indica a severidade da exposição à vibração; porém, não existe um consenso na comunidade científica da relação precisa entre VDV e o risco de ferimentos a que o corpo humano pode sofrer, sendo que durante o desempenho da atividade a campo valores de VDVs em torno de $15 \mathrm{~m} \mathrm{~s}^{-}$ ${ }^{1,75}$ usualmente podem ocorrer em superfíciesmuito acidentadas, causando severo desconforto, dores e ferimentos.

Para a projeção de 8 horas de trabalho os resultados encontrados repetem o cenário encontrado para o valor de dose de vibração, com a pista padronizada de ensaios de vibração apresentando as condições mais insalubres para o operador e o implemento acoplado ao trator auxiliando na amenização desta insalubridade (Tabela 5).

Tabela 5. Valores de projeção para jornada de 8 horas $\left(\mathrm{m} \mathrm{s}^{-2}\right)$ obtidos com o trator operando com e sem implemento em três condições superficiais distintas.

\begin{tabular}{cccc}
\hline & \multicolumn{3}{c}{ Superfície } \\
\hline Implemento & Concreto & Solo Mobilizado & Pista de Vibração \\
\hline Sem & $0,103 \mathrm{Ac}$ & $0,236 \mathrm{Ab}$ & $0,847 \mathrm{Aa}$ \\
Com & $0,089 \mathrm{Ac}$ & $0,157 \mathrm{Bb}$ & $0,731 \mathrm{Ba}$
\end{tabular}




\begin{tabular}{cc}
\hline F de Implemento & $303,64^{* *}$ \\
F de Pista & $11624,29^{* *}$ \\
F de Implemento x Pista & $55,66^{* *}$ \\
CV (\%) & 2,36 \\
DMS Linha & 0,018 \\
DMS Coluna & 0,015 \\
\hline
\end{tabular}

Médias seguidas de mesma letra maiúscula não diferem entre si na coluna e mesma letra minúscula não diferem entre si na linha pelo teste Tukey $(p \leq 0,05)$. ns: não significativo pelo teste $F$ ao nível de $5 \%$ de probabilidade. ${ }^{*}$ Significativo pelo teste $\mathrm{F}$ ao nível $5 \%$ de probabilidade. ${ }^{* *}$ Significativo pelo teste $\mathrm{F}$ ao nível de $1 \%$ de probabilidade.

Apenas os valores encontrados na pista padronizada de ensaio de vibração ficaram acima do nível de ação (faixa entre 0,5 e 0,9 $\mathrm{m} \mathrm{s}^{-2}$ ), o que implica na necessidade de intervenções com caráter preventivo para evitar possíveis lesões e problemas de saúde aos operadores, como adoção de menores velocidades de operação, treinamento e conscientização dos operadores sobre a importância da adoção de boas práticas de trabalho e da seleção correta das regulagens da máquina e implemento para operação.

Conhecer a ocorrência de condições insalubres geradas durante o desempenho das atividades agrícolas é importante para preservar a saúde do operador. Santos Filho et al. (2003), em experimento utilizando diferentes marchas para deslocamento do trator durante operação de gradagem sobre solo arado, obtiveram valores de aceleração ponderada global bem acima dos limites determinados para uma exposição de 4 horas de trabalho, de acordo com a Norma ISO 2631 (1997). Cunha et al. (2009), obtiveram resultados onde o limite para oito horas diárias de trabalho foi excedido, sendo que as piores situações ocorreram com as maiores acelerações ocorrendo no sentido de deslocamento do trator a medida que a rotação do motor foi aumentada.

Marsili et al. (2002), demostraram que a adoção de sistemas de suspensão pode permitir um incremento superior a $50 \%$ no tempo de exposição do operador ao trabalho. Lanças (2009) indica que, somente em condições específicas de operação com tratores agrícolas é possível atingir as oito horas diárias de trabalho. Segundo Tosin (2009), a vibração incidente sobre o operador está diretamente relacionada com a velocidade de deslocamento do trator, sendo que em seu experimento apenas as velocidades médias de $1,0 \mathrm{~m} \mathrm{~s}^{-1}$ e $1,4 \mathrm{~m} \mathrm{~s}^{-1}$ permitiram uma jornada completa de 8 horas de trabalho, em pista de concreto.

\section{CONCLUSÕES}

O ruído máximo interno da cabine permite que o operador desempenhe uma jornada de trabalho de 8 horas.

Somente foram geradas condições insalubres na pista padronizada de ensaio de vibração independentemente da presença de implemento acoplado ao sistema de três pontos do trator.

\section{REFERÊNCIAS BIBLIOGRÁFICAS}

ABRAHÃO, J.; SZNELWAR, L. I.; SILVINO, A.; SARMET, M.; PINHO, D. Introdução à Ergonomia: da Prática à Teoria. São Paulo: Edgard Blucher, 2009. 240 p. 
BAESSO, M. M.; GAZZOLA, M.; BERNARDES, S; BRANDELERO, E.; MODOLO, A. Avaliação do nível de ruído, itens de segurança e ergonomia em tratores agrícolas. Brazilian Journal of Biosystems Engineering. v.9(4): pp.368-380, 2015.

BALBINOT, A. Caracterização dos níveis de vibração em motoristas de ônibus: Um enfoque no conforto e na saúde. Tese (Doutorado em Biomecânica) - Universidade Federal do Rio Grande do Sul, Porto Alegre, $281 \mathrm{f}, 2001$.

BRAUNBECK, O. A.; ABRAHÃO, R. F.; GONZAGA, M. C. Protective gloves on manual sugar cane cutting are really effective?. A Journal of Prevention, Assessment and Rehabilitation, v. 41, p. 49634966, 2012.

CUNHA, J. P. A. R. DA; DUARTE, M. A. V.; RODRIGUES. J. C. Avaliação dos níveis de vibração e ruído emitidos por um trato $r$ agrícola em preparo de solo. Pesquisa Agropecuária Tropical, Goiânia, v. 39, n. 4, pp. 348-355, 2009.

DOIMO, L.S.; MORAES, G.A.G.; SANTOS, J.E.G., ANDREOLI, A.L.; Avaliação da vibração no posto operacional de tratores com baixa potência nominal no motor. vol. 2 num. pp. $1-15$. 2015.

EMBRAPA. Centro Nacional de Pesquisa de Solos. Sistema brasileiro de classificação de solos. Rio de Janeiro: Embrapa, 412p. 1999.

FRANCHINI, D. Análise do nível de vibrações verticais no assento de um trator agrícola. (Mestrado em Engenharia Agrícola) - Universidade Federal de Santa Maria, Santa Maria, 138 f, 2007.

FUNDACENTRO. Norma de higiene ocupacional: NHO 09: avaliação da exposição ocupacional a vibrações de corpo inteiro: procedimento técnico. São Paulo: Fundacentro, 2013. 63 p.

GERGES, S. N. Y. Ruído: Fundamentos e Controle. 2. ed. Florianópolis: Nr, 2000. 676 p.

IIDA, I. Ergonomia: Projeto e Prática. 2. ed. São Paulo: Edgard Blucher, 2005. 630 p.

INTERNATIONAL ORGANIZATION FOR STANDARDIZATION (ISO). 5008:2002. Agricultural wheeled tractors and field machinery - measurement of whole-body vibration of the operator.Geneva, Switzerland, 2002.

LANÇAS, K.P.; TOSIN, R. C.; ARAUJO, J. A. B.; MONTEIRO, L. DE A.; GUERRA, S. P. S. Avaliação da Vibração Ocupacional no Corpo Inteiro para Tratores Agrícolas de 55,2 kw e 109,5 kw. CONBEA, Juazeiro, BA/ Petrolina, PE, ago. 2009.

LANÇAS, K. P.. Elementos básicos para adequação de conjuntos mecanizados. In: MONTEIRO, L. A.; SILVA, P. R. A. Operação com tratores agrícolas. Botucatu: Edição dos Autores, 2009. p. 59-74.

MARSILI, A.; RAGNI, L.; SANTORO, G.; SERVADIO, P.; VASSALINI, G. Innovative Systems to reduce Vibrations on Agricultural Tractors: Comparative Analysis of Acceleration transmitted through the Driving Seat. Biosystems Engineering. UK. v. 81, pp: 35 - 47. 2002.

NORMA REGULAMENTADORA. NR 15: Atividades e Operações Insalubres. 12 ed. São Paulo: Saraiva, 2013.

PIMENTA JUNIOR, C. G.; DELMOND, J. G.; CUNHA, J. P. B.; COUTO, R. F.; LEONÍDIO, D. M.; DOS REIS, E. F. Análise espacial do nível de ruído emitido por trator agrícola. Revista Brasileira de Ciências Agrárias, vol. 7, núm. 3, pp. 514-520, 2012. 
ROSEIRO, M. N. V.; TAKAVANAGUI, A. M. M. Meio Ambiente e Poluição Atmosférica: O Caso da Cana-de-açúcar. Revista Saúde, v. 30, n. 2, p. 76-83, 2004.

SALIBA, T.M. Manual de Avaliação e Controle do Ruído. 3. ed. LTr. São Paulo. 2004. 110 p.

SANDI, J. Vibração incidente sobre o corpo inteiro do operador de trator agrícola ensaiado em pista de vibração com diferentes lastros, velocidades e pressões de inflação dos pneus. Dissertação (mestrado) - Universidade Estadual Paulista Júlio de Mesquita Filho, Faculdade de Ciências Agronômicas de Botucatu, 101 f, 2015.

SANTOS, L. N.; FERNANDES, H.C.; SOUZA, A.P.; JÚNIOR, M.R.F.; SILVA, R.M.F.; avaliação dos níveis de ruído de um conjunto trator-pulverizador, em função da velocidade de trabalho. Apresentado no XLIII Congresso Brasileiro de Engenharia Agrícola - CONBEA 201427 a 31 de julho de 2014- Campo Grande- MS, Brasil.

SANTOS FILHO, P. F. FERNANDES, A. C.; QUEIROZ, D. M. DE; SOUZA, A. P. DE; CAMILO, A. J. Avaliação dos níveis de vibração vertical no assento de um trator agrícola de pneus utilizando um sistema de aquisição automática de dados. Revista Árvore, Viçosa, v. 27, n. 6, pp.887-895, 2003.

SCARLETT, A. J., PRICE, J. S., STAYNER, R. M. Whole-body vibration: Evaluation of emission and exposure levels arising from agricultural tractors. Journal of Terramechanics. Silsoe - UK. v. 44. pp: 65-73. 2007.

SILVA, J.L.O; MIRANDA, L.K.M.; VIANA, F.P. percepção dos riscos ambientais de trabalhadores de uma usina sucroalcooleira. Estudos.Goiânia, v. 42, n. 4, p. 627-637, out/dez. 2015.

TOSIN, R. C.. Avaliação do ruído e da vibração no posto de trabalho em dois tratores agrícolas. Tese (Doutorado em Energia na Agricultura) - Faculdade de Ciências Agronômicas da UNESP, Campus de Botucatu, 140 f, 2009.

ZEHSAZ, M.; SADEGHI, M.H.; ETTEFAGH, M.M.; SHAMS, F.Tractor cabin's passive suspension parameters optimization via experimental and numerical methods. Journal of Terramechanics, Amsterdã, 2011. 48, 439-450. ISSN: 0022-4898.

\section{AGRADECIMENTOS}

Ao CNPQ pela oportunidade de aquisição dos equipamentos utilizados no ensaio, acelerômetro modelo HVM-100 e decibelímetro modelo DEC-300. A CAPES pelas bolsas concedidas aos alunos de pós-graduação autores deste trabalho. 